\title{
Distribución espacial y variables explicativas de capturas de Thunnus albacares (Perciformes: Scombridae) y especies no objetivo por la flota internacional de cerco en el Pacífico de Costa Rica
}

\author{
Priscilla Cubero-Pardo ${ }^{1}$, Juan B. Chavarría-Chaves ${ }^{2} \&$ Raquel Romero-Chaves ${ }^{3}$ \\ 1. Consejo Nacional de Rectores y Laboratorio PRIAS, Centro Nacional de Alta Tecnología, Pavas, San José, Costa \\ Rica; pcubero@conare.ac.cr \\ 2. Centro de Investigación en Ciencias del Mar y Limnología, San Pedro, San José, Costa Rica; chavarrj@yahoo.com \\ 3. Unidad de Investigación Pesquera y Acuicultura, Centro de Investigación en Ciencias del Mar y Limnología, San \\ Pedro, San José, Costa Rica; raquelromerochaves@gmail.com
}

Recibido 17-VII-2020. Corregido 02-XI-2020. Aceptado 26-XI-2020.

\begin{abstract}
Spatial distribution and correlates for catches of Thunnus albacares (Perciformes: Scombridae) and non-target species by the international purse-seine fleet in the Pacific of Costa Rica. Introduction: In the Pacific marine ecosystems of Costa Rica, fishing activity by the international purse-seine fleet is important for its wide scope and large impact. Objective: To evaluate the spatial distribution of the catch of yellowfin tuna and non-target species by this fleet, and possible correlates with environmental and fishing variables. Methods: We applied geospatial statistics and multiple regression models to Inter-American Tropical Tuna Commission data, covering from 2002 to 2011. We used a grid the size of the Exclusive Economic Zone (EEZ) with 1124 cells of $22 \times 22 \mathrm{~km}$ (unit of analysis), for a total of 11240 year-cells. Results: The medium tuna (presumably immature for the most part), dolphins, mantas and rays dominated catches within the Thermal Dome (Northeast of the EEZ). Small tuna (all immature), sharks, billfishes, dorado, wahoo, and various small species were caught mostly in the extreme South. Large tuna (mature) was caught specially in the zone center, towards the east of the Submarine Mounts Marine Management Area and the Isla del Coco National Marine Park. Tuna, dorado, dolphins, mantas and rays showed high site fidelity due to their association with geographic and environmental variables, at the time small tuna was associated with sets on floating objects, and big tuna was associated with sets on dolphins. The heterogeneity of non-target species probably hid most correlations. Conclusions: Fishing is affecting the highest trophic levels and an adjusted zoning design is needed to better protect the thermal dome and to take into account susceptibility to the use of purse seines.
\end{abstract}

Key words: bycatch; immature tuna; fisheries spatial analysis; floating objects; yellowfin tuna.

Cubero-Pardo, P., Chavarría-Chaves, J.B., \& Romero-Chaves, R. (2021). Distribución espacial y variables explicativas de capturas de Thunnus albacares (Perciformes: Scombridae) y especies no objetivo por la flota internacional de cerco en el Pacífico de Costa Rica. Revista de Biología Tropical, 69(1), 245-261. DOI 10.15517/rbt. v69i1.43005

La zonificación marina se refiere a un diseño dentro de un proceso de planificación espacial, que divide un área del mar en zonas dentro de las cuales los usos humanos son regulados. Apoya objetivos tanto económicos como sociales, así como un aprovechamiento sustentable de espacios y recursos, mediante la demarcación de áreas clave a ser conservadas, dentro de un proceso público y político (Ehler \& Douvere, 2013). Constituye una herramienta de manejo como base para la implementación de un plan general y sub-planes derivados, y es representada por un mapa que demarca las zonas, cada una vinculada con una serie de 
regulaciones (GBRMPA, 2004). El manejo de grandes áreas marinas mediante zonificación dentro de procesos de planificación espacial se encuentra en diversos estados de avance en el mundo, y permite minimizar conflictos entre sectores, sub-sectores y usuarios (Paxinos et al., 2008; Castrejón \& Charles, 2013; Dineshbabu, Sujitha, Prathibha, \& Maheswarudu, 2019).

Como base para crear las zonas, y corroborar su efectividad a lo largo del tiempo, se requiere contar con datos que permitan comprender la dinámica de los recursos y los requerimientos de los usuarios, a partir de un sistema con base científica, socialmente sensible y adaptativo, basado en monitoreo (26/MAR/2020, URL: https://floridakeys.noaa. gov/about/welcome.html?s=about). Esto es clave, dada la naturaleza móvil de los recursos marinos, eventuales cambios en las necesidades humanas, y la posibilidad de variación en las condiciones ambientales que conlleven a la migración o modificación de áreas de distribución de las especies objetivo dentro de zonas específicas (Sanchirico, 2000).

Estudios previos indican que, para algunas zonas, pueden declararse medidas de protección para recursos o hábitats, y permitir pocos usos compatibles o ningún uso, según el nivel de protección deseado. Para otras zonas, sin embargo, la protección de recursos puede ser menos prioritaria y permitir usos más o menos intensivos, siempre considerando la capacidad de los recursos para soportar tales usos sin sufrir desequilibrio (Courtney \& Wiggin, 2003; Klein, Steinback, Watts, Scholz, \& Possinham, 2009).

En el caso del diseño de herramientas de zonificación de pesquerías es indispensable contar con amplias bases de datos pesqueros georreferenciados, incluyendo captura de especies no objetivo (pesca incidental), y conocer la afinidad de las flotas con determinados recursos (Meaden, Aguilar-Manjarrez, Corner, O'Hagan, \& Cardia, 2016). Todo lo anterior, con el fin de satisfacer metas tanto económicas como sociales e identificar zonas ambientalmente sensibles, en procura de la preservación de las especies (Arriaga \& Martínez, 2003; Dineshbabu et al., 2019).

Dentro del manejo pesquero, una parte esencial es la zonificación espacial, a través de la declaración de áreas según el tipo de pesquería y arte de pesca, ya que contribuye a delimitar el alcance espacial de las distintas flotas e imponer límites de uso en condiciones de alta fragilidad (p.e. concentración de especies no objetivo y criaderos), entre otros aspectos (Klein et al., 2009). La falta de manejo pesquero lleva inevitablemente a la sobreexplotación de los recursos y a pérdida de hábitats (Meaden et al., 2016), como ha sido el caso del atún aleta azul del Atlántico Occidental (Thunnus thynnus), cuya negligencia en la aplicación de medidas de manejo recomendadas ha llevado a esta especie a una declinación preocupante (Safina, 1993).

De acuerdo con Jiménez (2013), en Costa Rica la pesca de tiburones, marlines, velas y dorado dio inicio en la década de 1990, con declinación significativa en las capturas una década después. Actualmente, la declinación continúa, con los tiburones representando el $40 \%$ de las capturas mar adentro. Esas especies son blanco de los sectores palangrero y de pesca turística y deportiva nacionales, por lo que falta de planificación y manejo de sus pesquerías conlleva a conflictos entre ambos sectores. La flota de cerco, desarrollada exclusivamente por barcos de bandera extranjera, captura incidentalmente una alta cantidad de dorado y picudos, y se convierte en una tercera fuente de conflicto por esas especies.

Esa flota también genera alta captura incidental de diversas especies incluyendo delfines, tortugas, mantas, rayas, tiburones y otras de pequeño tamaño (Hall \& Boyer, 1986; Hall \& Roman, 2013; Croll et al., 2015; Lezama-Ochoa et al., 2016; Lezama-Ochoa, Hall, Román, \& Vogel, 2019). Por ese motivo, también entra en conflicto con los sectores de turismo marino y de pesca artesanal, debido a competencia por esos recursos, y afecta negativamente a las flotas que utilizan artes de pesca selectivas (p.e. pesca con línea de fondo o con palo verde). 
En el Océano Pacífico Oriental, la pesca con red de cerco ha producido incrementos en la mortalidad por pesca de atunes patudos jóvenes (Thunnus obesus) (Watters \& Maunder, 2001). Además, ha tenido impacto sobre la biomasa reproductora del atún aleta amarilla (T. albacares) (Minte-Vera, Maunder, \& Aires-da-Silva, 2018). El uso de dispositivos agregadores de peces (o plantados), como arte complementario, intensifica la captura incidental de diversas especies y genera alta captura de atunes jóvenes (Castro, Santiago, \& Hernández-García, 1999; Hallier \& Gaertner, 2008; Morgan, 2011). La falta de selectividad del uso de red de cerco pone en riesgo la sostenibilidad de la propia pesquería a largo plazo (Torres-Irineo et al., 2014).

Un análisis previo de los datos utilizados en el presente estudio, basado exclusivamente en estadística descriptiva, reveló un amplio alcance espacial de la actividad pesquera por la flota de cerco en el Pacífico de Costa Rica (Cubero-Pardo \& Martínez-Cascante, 2013). El presente trabajo explora los efectos de dicha flota sobre los ecosistemas marinos del Pacífico costarricense, a fin de dilucidar aspectos clave que pueden contribuir a mejorar la planificación en el uso del espacio marino, para beneficio de esos ecosistemas y de actividades económicas, con base en dos objetivos: (1) evaluar la distribución espacial de la captura por unidad de esfuerzo (CPUE) del atún aleta amarilla, Thunnus albacares, y de otras especies pelágicas, utilizando análisis estadísticos geoespaciales, y (2) analizar la captura estandarizada, en función de variables ambientales y pesqueras que pueden explicar dicha captura, mediante la aplicación de modelos de regresión.

Se asume que es posible diferenciar áreas donde la CPUE es significativamente alta en cada caso analizado y que las áreas de distribución de las capturas observadas, tanto para el atún aleta amarilla, como para otras especies pelágicas, están asociadas a determinadas condiciones ambientales y pesqueras. Los resultados se discuten en función de su contribución a la planificación espacial de pesquerías en el Pacífico de Costa Rica, y de la importancia de identificar áreas clave para ser protegidas o manejadas.

\section{MATERIALES Y MÉTODOS}

Área de Estudio: Abarca toda la Zona Económica Exclusiva (ZEE) del Pacífico de Costa Rica, la cual cubre $543842 \mathrm{~km}^{2}$ (Fonseca, 2006). A partir de octubre de 2014, fue oficializada una zonificación espacial de pesquerías para toda la ZEE ubicada fuera de las 20 áreas marinas protegidas (Salas, Salazar, \& Arias, 2012) y de las Áreas Marinas de Pesca Responsable existentes, bajo un decreto de "Ordenamiento para el aprovechamiento de atún y especies afines en la zona económica exclusiva del Océano Pacífico costarricense" (Decreto 38681 MAG-MINAE, La Gaceta No. 213, 05 de noviembre 2014).

Dicha zonificación definió cinco zonas (Fig. 1), bajo los siguientes usos: (I) para uso de las flotas de pesca comercial Mediana Escala y Avanzada, pesca Turística y Deportiva y pesca comercial Semi-Industrial, (II) definida como un espacio de transición entre el polígono costero y el resto del entorno marino, para amortiguar el impacto directo de la pesca con mayor capacidad tecnológica, (III) y (IV) permiten únicamente la pesca comercial avanzada que utilice como arte de pesca el palangre con línea madre de monofilamento, así como la pesca con caña, curricán y cuerda de mano, siempre y cuando se utilice anzuelo circular. La zona (IV) se considera una zona de reclutamiento de atún y especies afines, dirigida al aprovechamiento sostenible de los recursos pesqueros. La zona (V) permite el uso por parte de barcos de cerco de bandera extranjera, así como las flotas costarricenses.

Datos: Continuamente, a partir de las faenas de pesca realizadas por las embarcaciones de cerco de bandera extranjera, la Comisión Interamericana del Atún Tropical (CIAT) levanta una base de datos georreferenciada con registro de cada lance realizado. En cada lance son registrados datos de referencia (i.e. capacidad de bodega del barco, número de viaje, de 


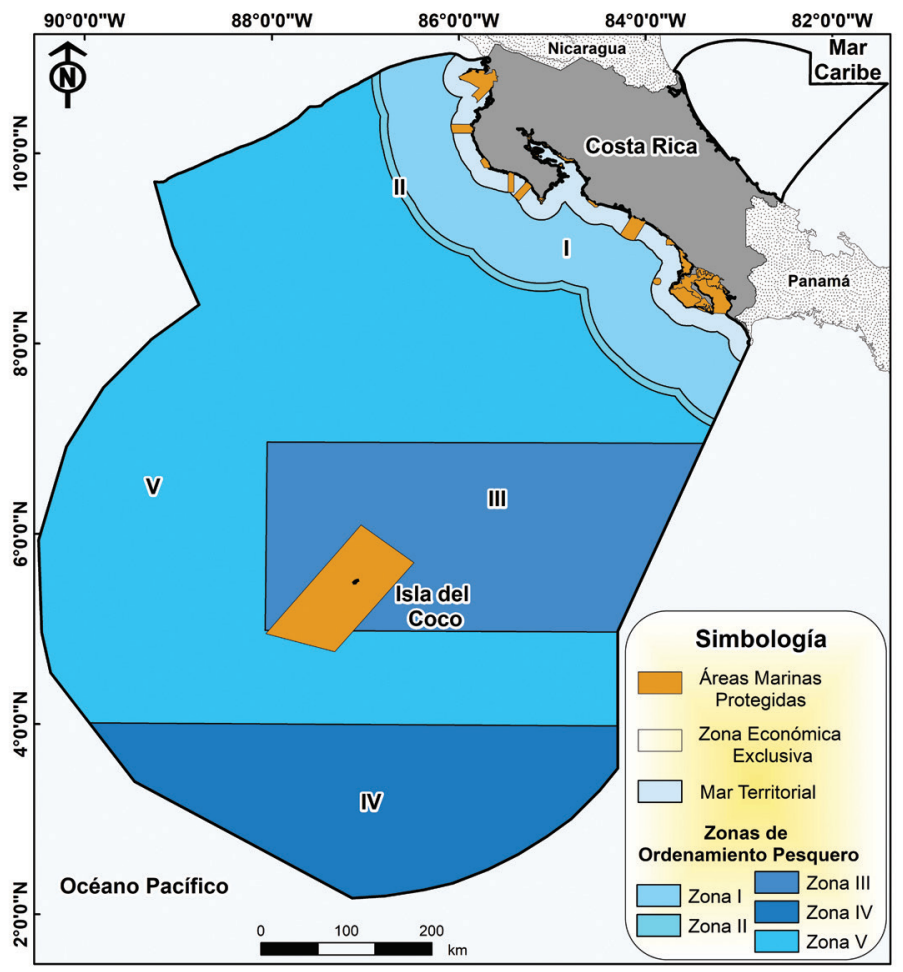

Fig. 1. Diseño de la zonificación de pesquerías decretada para el Pacífico costarricense, en octubre 2014.

Fig. 1. Design of the fisheries zoning decreed for the Costa Rican Pacific, in October 2014.

lance y posición geográfica), datos temporales (i.e. año), datos de captura (toneladas métricas por especie de atún o por especie no objetivo) y datos de clasificación (i.e. tipo de lance, grupos de peso por especie de atún).

Este estudio analiza datos georreferenciados recolectados por la CIAT entre 2002 y 2011, organizados en dos hojas electrónicas, las cuales involucran: (a) 13349 registros de lances para captura de atún, clasificando el producto por clases de peso y (b) 8648 registros de lances para captura de atún, asociados a captura incidental de especies no objetivo de esta pesquería. Ambas hojas están relacionadas por un número de identificación de lance $\mathrm{y}$, en cada una, se indica, para cada lance, variables asociadas (Apéndice digital 1).

En este trabajo, los análisis referentes al atún por clase de peso son centrados exclusivamente en el atún aleta amarilla (11 692 registros de lances), porque a esta especie correspondió el $87.60 \%$ de capturas, entre seis especies (Apéndice digital 2). A las dos hojas de datos analizadas fueron adicionados datos georreferenciados de profundidad, temperatura superficial del mar y clorofila, obtenidas de fuentes de datos satelitales en línea (Apéndice digital 3).

Los datos de peso de atún aleta amarilla, fueron originalmente clasificados en tres categorías en la base de la CIAT (Apéndice digital 1), y como tales se utilizan en este estudio, separadas en tres nuevas hojas de datos. Cada lance realizado pudo contener captura de una sola clase de peso, dos clases o de las tres clases. Debido a que existe un mínimo legal de captura de esta especie, a partir de $3.4 \mathrm{~kg}$ de peso, cuando ya se registran individuos sexualmente maduros en la población (Davidoff, 1963), este estudio asume que la categoría de peso bajo $(<2.5 \mathrm{~kg})$, o atún pequeño, corresponde a individuos sexualmente inmaduros; que la categoría de peso medio $(2.5 \mathrm{a}<15 \mathrm{~kg})$, o atún 
mediano, contiene tanto individuos inmaduros como maduros, y que la categoría de peso alto ( $>15 \mathrm{~kg}$ ), o atún grande, refiere a individuos sexualmente maduros. En el peso mediano no es posible conocer si existe proporción dominante de organismos maduros o inmaduros entonces, bajo el Principio Precautorio, este estudio considera los pesos bajo y mediano como individuos sexualmente inmaduros.

Por su parte, los datos de las especies no objetivo fueron clasificados en siete categorías taxonómicas, y a cada una se le asignó valor preponderante para actividades económicas particulares (Tabla 1). Estas categorías fueron segregadas en siete nuevas hojas de datos.

Unidad de análisis: Los datos de captura, asociados a coordenadas $\mathrm{x}$ e $\mathrm{y}$, fueron vinculados a una grilla del tamaño de la ZEE del Pacífico de Costa Rica, conformada por 1124 celdas de 22 × $22 \mathrm{~km}$, como unidad de análisis. En el periodo de 10 años cubiertos por los datos, las 1124 celdas mencionadas se convirtieron en 11240 celdas-año. Sin embargo, no en todos los años ocurrió captura de atún o captura de especies no objetivo en cada cuadrícula, y hubo cuadrículas sin presencia de captura a lo largo de la década. Por ese motivo, el número de celdas-año sometidas al análisis varió en las diez hojas de datos generadas para los análisis. A partir de cada hoja de datos, se construyeron matrices, las cuales fueron utilizadas para aplicar, por un lado, análisis de puntos calientes y, por otro, análisis de regresión múltiple, utilizando un modelo lineal generalizado normal identidad (MLG-NI), un modelo lineal generalizado normal logarítmico (MLG-NL) y un modelo lineal generalizado gamma logarítmico (MLG-GL).

El tamaño de celda de $484 \mathrm{~km}^{2}$ se consideró óptimo para representar visualmente los patrones espaciales de captura en el área de estudio, tomando en cuenta la resolución de los datos y el ajuste del modelo de regresión (Riolo, 2006).

Análisis de puntos calientes: Este análisis fue realizado con el uso de varias herramientas de ArcMap 10.7.1. Como primer paso, fueron eliminados los valores extremos de cada base de datos, con el uso de la herramienta Análisis Optimizado de Valores Extremos. En cada base resultante, fue identificada la distancia a la cual cada dato tenía ocho vecinos, usando la distancia máxima generada por la herramienta $\mathrm{Calcu}$ lar Banda De distancia de Conteo de Vecinos.

Seguidamente, fue definida la distancia a la cual se presentaba la máxima autocorrelación de los datos (óptima), mediante el cálculo del Índice de Moran Global (I de Moran), con el uso de la herramienta Autocorrelación Espacial Incremental (AEI), con base en las

TABLA 1

Categorías taxonómicas de especies marinas no objetivo capturadas por la flota de cerco en el Pacífico de Costa Rica, de 2002 a 2011, con total de especies, registros, toneladas métricas (TM) capturadas y valor económico

TABLE 1

Taxonomic categories of non-target marine species caught by the purse-seine fleet in the Pacific of Costa Rica, from 2002 to 2011, with total species, records, metric tons (MT) caught and economic value

\begin{tabular}{llccc}
\multicolumn{1}{c}{ Categorías } & \multicolumn{1}{c}{ Valor económico } & Total de especies & Registros & TM \\
Delfines & Turismo marino & 6 & 374 & 55.17 \\
Mantas y rayas & Turismo marino & 6 & 1143 & 338.61 \\
Tiburones & Pesca extractiva / Turismo marino & 12 & 1012 & 196.64 \\
Picudos & Pesca extractiva & 6 & 1808 & 342.52 \\
Dorado & Pesca turística & 2 & 918 & 192.71 \\
Peto & Pesca deportiva & 1 & 717 & 46.31 \\
Especies diversas & Pesca extractiva / Turismo marino & 16 & 737 & 197.55 \\
TOTAL & & 49 & 6709 & 1369.50 \\
\hline
\end{tabular}


coordenadas geográficas de cada celda junto con la CPUE. Para correr la AEI, fueron elegidas 30 bandas de distancia, fue utilizada la distancia de los ocho vecinos identificada previamente como 'distancia de inicio', y fueron probadas varias 'distancias de incremento'. La AEI calcula varios valores del I de Moran, el cual establece los patrones en los datos dentro de un ámbito entre -1 (patrón disperso) y 1 (patrón agrupado), con valores cercanos a cero indicando una distribución aleatoria (Moran, 1950; Jalali, Lerodiaconou, Gorfine, Monk, \& Rattray, 2015). A su vez, el I de Moran utiliza una medida de significancia estadística basa$\mathrm{da}$ en una puntuación $\mathrm{Z}$ y un valor $\mathrm{P}$, donde valores positivos de $\mathrm{Z}(>1.65, \mathrm{P}<0.10)$ indican un patrón agregado, mientras que valores negativos de $\mathrm{Z}(<-1.65, \mathrm{P}<0.10)$ indican un patrón disperso (Ord \& Getis, 1995; Ord \& Getis, 2001; Abbott \& Haynie, 2012; e Costa, Gonçalves \& Gonçalves, 2013). En la AEI, la distancia óptima corresponde a aquella en la cual la puntuación $Z$ alcanza su máximo valor.

Por medio de la herramienta Análisis de Puntos Calientes (ArcMap v10.7.1), utilizando la captura por unidad de esfuerzo (CPUE, captura observada dividida entre número de lances) para cada cuadrícula-año, se identificaron agrupaciones espaciales estadísticamente significativas con alta tasa de captura (puntos calientes), y con baja tasa de captura (puntos fríos), tanto para el atún aleta amarilla, en las tres clases de peso, como para las especies no objetivo, clasificadas en las siete categorías taxonómicas (Tabla 1). Para este análisis, fueron excluidas las cuadrículas sin presencia de captura, en cada base de datos.

La herramienta Análisis de Puntos Calientes utilizó el algoritmo Getis-Ord Gi* (Ord \& Getis 1995; Ord \& Getis, 2001; Abbott \& Haynie, 2012; e Costa et al., 2013), con base en la distancia óptima definida por la AEI, para asignar un valor estadístico Gi* (puntuación Z) y un valor de $\mathrm{P}$ a cada una de las celdas. Así, fueron determinadas áreas con valores altos y bajos de captura estandarizada, con autocorrelación local y dependencia entre celdas vecinas estadísticamente significativas.
Los puntos calientes se detectaron cuando los valores de celdas adyacentes fueron $\mathrm{Gi}^{*}$ $\geq 1.65, \mathrm{P}<0.10, \mathrm{Gi}^{*} \geq 1.96, \mathrm{P}<0.05$ y Gi* $\geq 2.58, \mathrm{P}<0.01$. Un valor positivo de $\mathrm{Gi}^{*}$ asociado a un valor $\mathrm{P}<0.10$, indicó un punto caliente en las tasas de captura, mientras un valor negativo de $\mathrm{Gi}^{*}$ asociado a un valor $\mathrm{P}<$ 0.10 indicó un punto frío de tasas de captura. Las agrupaciones significativas se compararon con la hipótesis nula de aleatoriedad espacial completa $(C S R)$.

A partir de los resultados de los análisis de puntos calientes, para las tres hojas de datos de atún clasificado por clase de peso, fueron extraídas las áreas de agregación significativa de las capturas, a un $99 \%$ de confianza ( $\mathrm{Gi}^{*}$ $\geq 2.58, \mathrm{P}<0.01$ ), mientras que para las siete hojas de datos de especies no objetivo fueron extraídas las áreas de agregación significativa de las capturas, a un $90 \%$ de confianza (Gi* $\geq$ 1.65, $\mathrm{P}<0.10)$. En todos los casos, los valores $\mathrm{Gi}^{*}$ fueron agrupados con base en una escala de 10 niveles utilizando la clasificación de Quiebres Naturales (Jenks), con el fin de identificar zonas con mayor a menor CPUE.

Este procedimiento para el análisis de puntos calientes fue aplicado, en forma adicional, a datos referentes a lances sobre delfines y sobre objetos flotantes (incluyó: objetos naturales, plantados y objetos no diferenciados entre naturales y plantados), a un $90 \%$ de confianza, como información adicional que permita visualizar la intensidad de uso de cada tipo de lance en la ZEE. Como las frecuencias fueron expresadas en porcentajes, no se censuró por valores extremos, ya que cero y cien son valores válidos en esta escala.

Estandarización de las capturas mediante regresión: Los valores observados de captura de atún aleta amarilla, clasificados por clase de peso, y de especies pelágicas no objetivo, clasificadas por grupo taxonómico, fueron estandarizados mediante regresión con modelo lineal generalizado (MLG) como se mencionó antes. El componente sistemático del modelo se planteó según la expresión (B) que se indica más adelante. Para esto se utilizaron variables 
de ubicación del lance (latitud*longitud), variables ambientales de temperatura superficial del mar, clorofila-a y profundidad (calculando valores promedio anuales para cada celda, Apéndice digital 3), y el tipo de lance como variable categórica (calculando porcentajes), clasificada en dos categorías: 'lance sobre delfines' y 'lance sobre objeto flotante'. La segunda categoría de tipo de lance integró datos referentes a objeto natural, plantado y objeto flotante (no definido entre natural y plantado) de las bases originales.

$$
\begin{gathered}
\mathrm{Y}^{\prime}=\mathrm{Bo}+\mathrm{B} 1^{*} \mathrm{f}+\mathrm{B} 2 * \text { Temperatura }+ \\
\mathrm{B} 3^{*} \text { Clorofila }+\mathrm{B} 4 * \text { Profundidad }+\ldots \text { (B) }
\end{gathered}
$$

Donde,

$Y^{\prime}=$ Captura predicha

$B_{0}=$ Coeficiente de intersección

$B_{1}, B_{2} \ldots=$ Coeficiente de regresión por variable

$f=$ Esfuerzo en número de lances

Mediante análisis exploratorio de datos se determinó que el componente aleatorio del modelo (la variable respuesta), expresado como captura en peso por celda, tiene una distribución de frecuencias muy asimétrica (como es común en pesquería) que se asemeja a una distribución de probabilidades Normal logarítmica (log-normal) o quizá exponencial (caso particular de una distribución Gamma) (Mendenhall, 1990). Esto llevó a sugerir el uso de una función de enlace logarítmica tanto para el modelo con la Normal como para el modelo con la distribución Gamma. También se usó la función de enlace identidad (sin transformación) con la Normal, por su uso tan difundido y alcance generalizado para ligar una respuesta con su predictor lineal (Agresti, 2002).

A través de contraste de hipótesis de Wald sobre los coeficientes de regresión, se identificaron las variables explicativas que más contribuían en cada modelo estimado. Además, se evaluó el nivel de ajuste de los modelos, mediante exploración de gráficos de los residuos y de los predichos, además de considerar los índices de Akaike (AIC) y bayesiano (BIC).
Previo a la aplicación de los análisis de regresión múltiple, fueron aplicados indicadores de censura al $2 \%$ (uno por ciento a cada lado del ámbito de variación de los datos) a las variables continuas, a nivel de cuadrícula, con el fin de identificar valores extremos. $\mathrm{Al}$ igual que en el análisis de puntos calientes, la variable categórica referente al tipo de lance no tuvo censura, pues sus valores extremos (cero, cien) son válidos.

\section{RESULTADOS}

Análisis de Puntos Calientes: La Autocorrelación Espacial Incremental (AEI) reveló patrones agregados (I de Moran positivo), al $1 \%$ de significancia $(Z>2.58, P<0.01)$ para las tres clases de peso de atún, y al $10 \%$ de significancia $(\mathrm{Z}>1.65, \mathrm{P}<0.10)$ para las agrupaciones de especies no objetivo, a excepción del peto y las especies diversas.

En el caso del peto, la AEI reveló un patrón general disperso para las capturas (I de Moran negativo) y, en el caso de las especies diversas, la herramienta no encontró una distancia óptima y, por tanto, no fue posible dilucidar un patrón espacial estadísticamente significativo para los datos (Tabla 2). Por ese motivo, el Análisis de Puntos Calientes para las especies diversas fue aplicado utilizando una distancia establecida por defecto por la herramienta, la cual aseguró que cada dato tuviera, al menos, un vecino.

Con base en los Análisis de Puntos Calientes, cada clase de peso de atún aleta amarilla y cada agrupación de fauna no objetivo presentó áreas claramente definidas de alta captura por unidad de esfuerzo (CPUE). En el caso del atún aleta amarilla fueron evidentes patrones de distribución claramente diferenciables entre las tres clases de peso (Fig. 2).

La captura más alta del atún pequeño se presentó en el extremo sureste, bordeando el límite de la Zona Económica Exclusiva (ZEE), con una cobertura de $103530 \mathrm{~km}^{2}$ (19.97\%); el atún mediano fue mayormente capturado en el extremo noroeste, con alta coincidencia con el Domo Térmico, en un área de $210424 \mathrm{~km}^{2}$ 
TABLA 2

Índice de Moran, Puntuación $Z$ y Valor P, derivados de la AEI, con base en la distancia óptima, para el atún y las especies no objetivo, y número de celdas cubiertas por las capturas en cada caso

TABLE 2

Moran Index, Z Score and P Value, derived from the AEI, based on the optimal distance, for tuna and non-target species, and number of cells covered by catches in each case

\begin{tabular}{|c|c|c|c|c|c|c|}
\hline & & Distancia (m) & Índice de Moran & Puntuación Z & $P$ & Celdas \\
\hline \multirow{3}{*}{ 焉 } & Pequeño & 394269 & 0.051639 & 31.704083 & 0.000000 & 5311 \\
\hline & Mediano & 449194 & 0.075056 & 250.087363 & 0.000000 & 4615 \\
\hline & Grande & 328433 & 0.041667 & 114.700070 & 0.000000 & 4942 \\
\hline \multirow{7}{*}{ 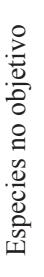 } & Delfines & 227370 & 0.230620 & 3.502491 & 0.000461 & 374 \\
\hline & Mantas y Rayas & 208000 & 0.006406 & 9.034038 & 0.000000 & 959 \\
\hline & Tiburones & 168314 & 0.014770 & 3.168048 & 0.001535 & 1012 \\
\hline & Picudos & 483000 & 0.038077 & 75.809393 & 0.000000 & 1672 \\
\hline & Dorado & 537000 & 0.020933 & 21.666034 & 0.000000 & 779 \\
\hline & Peto & 312000 & 0.027892 & 12.756944 & 0.000000 & 685 \\
\hline & Especies diversas & - & - & - & - & 737 \\
\hline
\end{tabular}
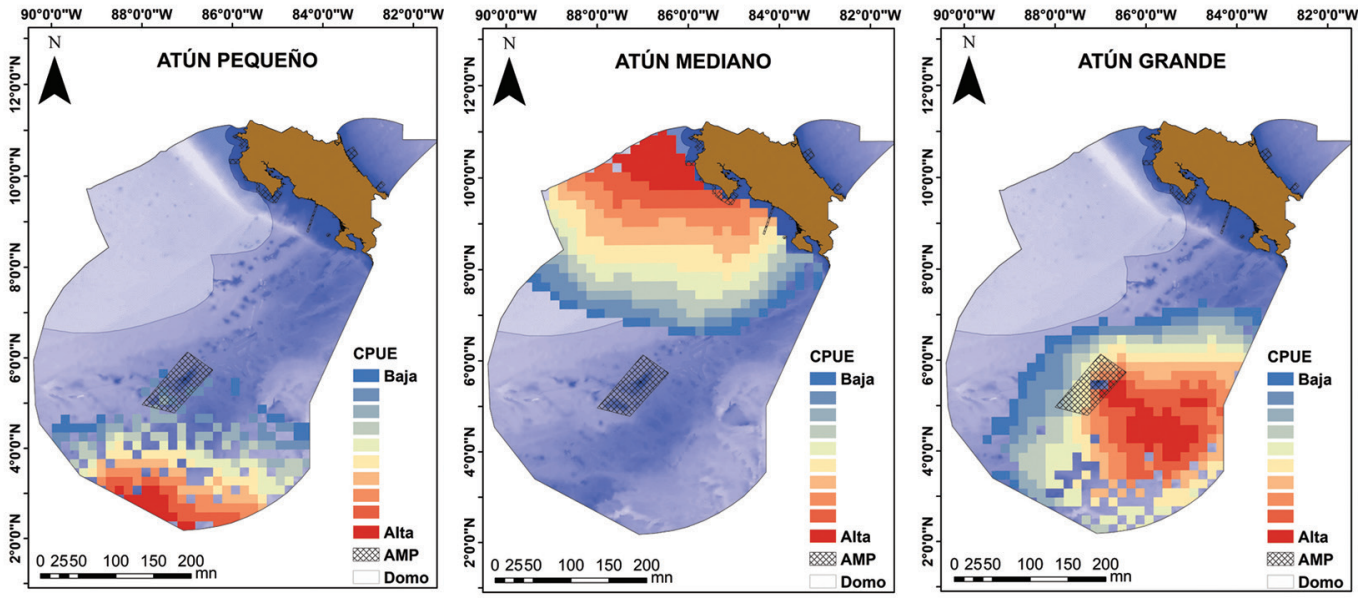

Fig. 2. Distribución de las áreas de mayor CPUE de atún aleta amarilla, Thunnus albacares, por clase de peso, capturado dentro de la Zona Económica Exclusiva del Pacífico de Costa Rica, del 2002 al 2011, por la flota de cerco. Capa del Domo Térmico: Fundación MarViva. 2016. Ubicación media estimada del Domo Térmico.

Fig. 2. Distribution of the areas with the highest CPUE of yellowfin tuna, Thunnus albacares, by weight class, caught within the Pacific Exclusive Economic Zone of Costa Rica, from 2002 to 2011, by the purse seine fleet. Thermal Dome Layer: MarViva Foundation. 2016. Estimated average location of the Thermal Dome.

(38.69\%), y el atún grande tuvo mayores capturas en un área de $224258 \mathrm{~km}^{2}(41.26 \%$ ), ubicada en el centro-sureste de la ZEE, rodeando el Área Marina de Manejo Montes Submarinos y el Parque Nacional Isla del Coco.

En la fauna no objetivo, los delfines y las mantas y rayas presentaron la mayor CPUE dentro de la zona del Domo Térmico, mientras los tiburones, los picudos, el dorado, el peto y las especies diversas, fueron capturados mayormente en el extremo sureste, coincidiendo con el atún pequeño (Fig. 3).

Para cada agrupación de fauna no objetivo, las zonas de mayor captura tuvieron la siguiente cobertura: delfines: $22988 \mathrm{~km}^{2}$ (4.23\%), mantas y rayas: $30161 \mathrm{~km}^{2}(19.04 \%)$, tiburones: 

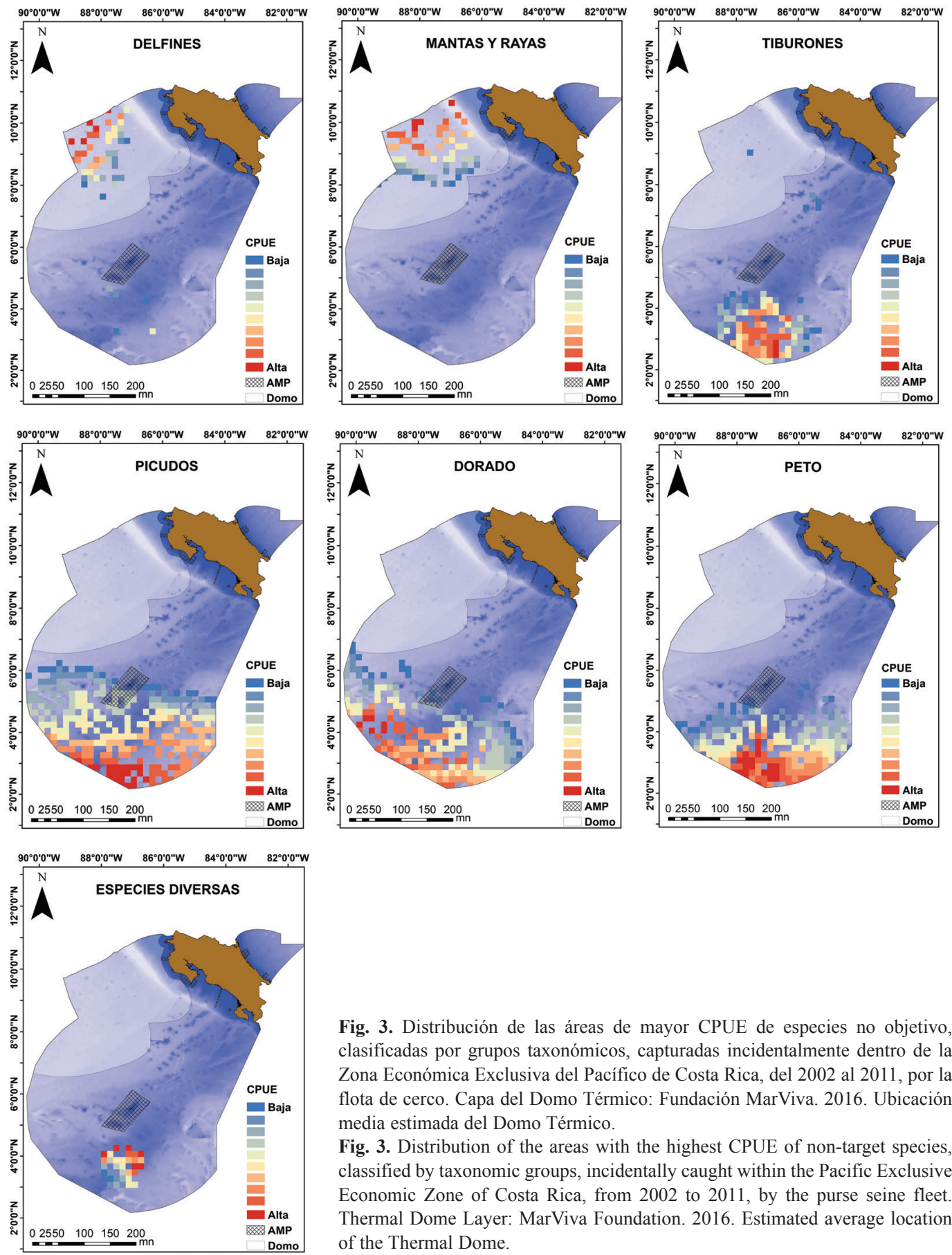

Fig. 3. Distribución de las áreas de mayor CPUE de especies no objetivo, clasificadas por grupos taxonómicos, capturadas incidentalmente dentro de la Zona Económica Exclusiva del Pacífico de Costa Rica, del 2002 al 2011, por la flota de cerco. Capa del Domo Térmico: Fundación MarViva. 2016. Ubicación media estimada del Domo Térmico.

Fig. 3. Distribution of the areas with the highest CPUE of non-target species, classified by taxonomic groups, incidentally caught within the Pacific Exclusive Economic Zone of Costa Rica, from 2002 to 2011, by the purse seine fleet. Thermal Dome Layer: MarViva Foundation. 2016. Estimated average location of the Thermal Dome. 

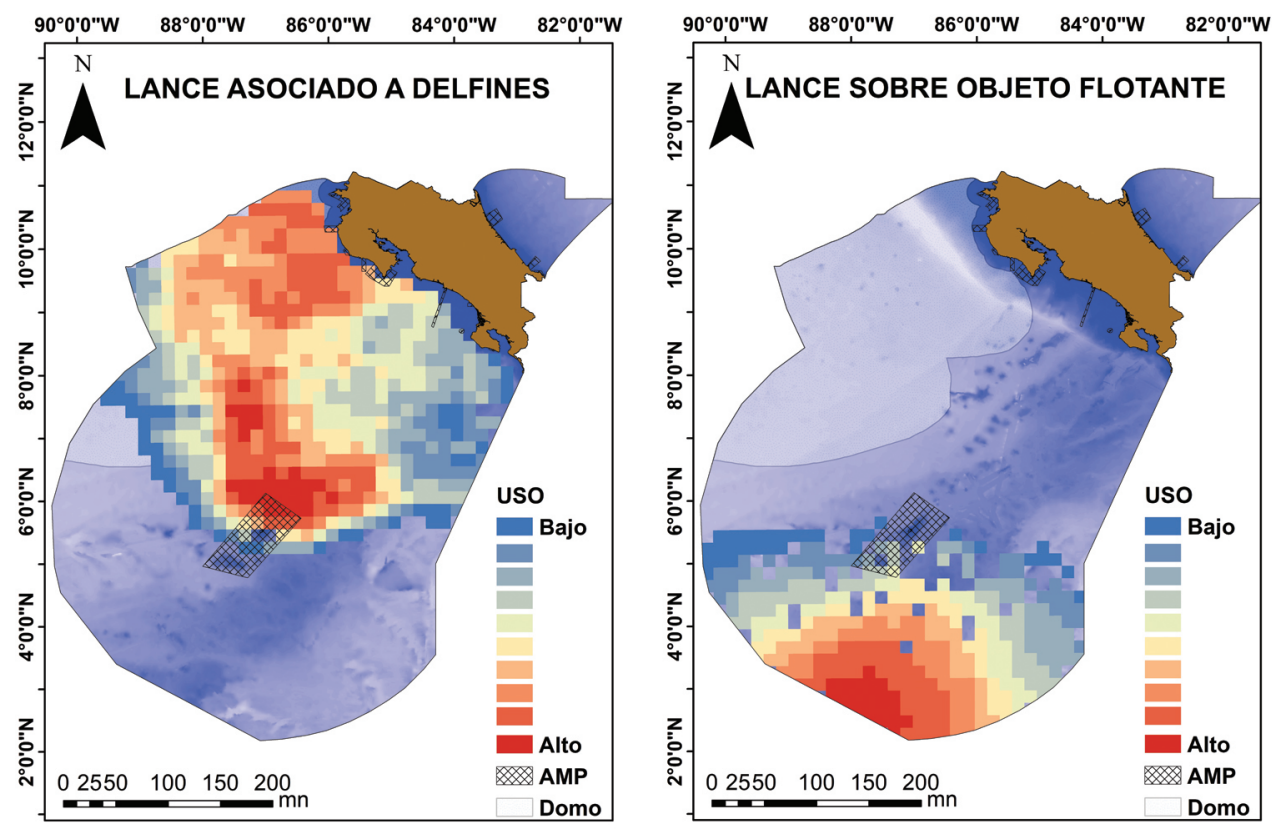

Fig. 4. Distribución de las áreas de mayor ejecución de lances asociados a objetos flotantes y a delfines, dentro de la Zona Económica Exclusiva del Pacífico de Costa Rica, del 2002 al 2011, por la flota de cerco. Capa del Domo Térmico: Fundación MarViva. 2016. Ubicación media estimada del Domo Térmico.

Fig. 4. Distribution of the areas with the highest execution of sets associated with floating objects and dolphins, within the Exclusive Economic Zone of the Pacific of Costa Rica, from 2002 to 2011, by the purse-seine fleet. Thermal Dome Layer: MarViva Foundation. 2016. Estimated average location of the Thermal Dome.

$49659 \mathrm{~km}^{2}$ (9.13\%), picudos: $126380 \mathrm{~km}^{2}$ (23.24\%), dorado: $101535 \mathrm{~km}^{2}(18.67 \%)$, peto: $94575 \mathrm{~km}^{2}(17.39 \%)$, especies diversas: $16456 \mathrm{~km}^{2}(3.03 \%)$.

Por su parte, los lances sobre delfines fueron realizados especialmente en el centro y en el extremo noroeste de la ZEE, sobre el área del Domo Térmico, con más alto uso en un área de $300010 \mathrm{~km}^{2}(55.1 \%)$, y los lances asociados a objetos flotantes fueron concentrados mayormente en el centro del extremo sur, con una cobertura de más alto uso en un área de 161107 $\mathrm{km}^{2}(29.63$ \%) (Fig. 4).

Estandarización de las capturas: Los modelos de regresión revelaron, a un nivel de $95 \%$ de confianza, la ubicación (lat*long), el número de lances, la clorofila, la temperatura, la profundidad, los lances sobre delfines y sobre objetos flotantes como variables significativas que explican la captura estandarizada del atún pequeño (MLG-NL); la ubicación, el número de lances, la clorofila y la profundidad como variables significativas que explican la captura estandarizada del atún mediano (MLG-NL), y la ubicación, el número de lances, los lances sobre delfines y sobre objetos flotantes, como variables significativas que explican la captura estandarizada para atún grande (MLG-NL) (Apéndice digital 4A, Apéndice digital 4B y Apéndice digital 4C).

Para las categorías de especies no objetivo, los modelos de regresión revelaron, a un $95 \%$ de confianza, el número de lances y los lances sobre delfines como variables que explican la captura estandarizada para los tiburones (MLG-GL), y el número de lances, la latitud y la temperatura como variables explicativas para dicha captura en las mantas y rayas (MLG-GL) y los picudos (MLG-GL); los últimos también 
fueron explicados por los lances sobre delfines. En el caso de los delfines (MLG-GL), el número de lances y la temperatura superficial del mar, fueron variables con asociación estadísticamente significativa a la captura; en el caso del dorado (MLG-NL), fueron la profundidad y los lances sobre delfines; para el peto (MLG-GL), se trató del número de lances, la latitud y los lances sobre objetos flotantes, y en el caso de las especies diversas (MLG-NL), correspondió al número de lances, la latitud, la profundidad, los lances sobre delfines y sobre objetos flotantes (Apéndice digital 4D, Apéndice digital 4E, Apéndice digital 4F, Apéndice digital 4G, Apéndice digital 4H, Apéndice digital 4I, Apéndice digital 4J).

\section{DISCUSIÓN}

Desde 2005, la política Estatal costarricense promueve bajo su Ley 8436 (La Gaceta No. 78, 25 de abril 2005), “...la protección de la biomasa pesquera para determinar el uso, el aprovechamiento sostenible, la ordenación, el manejo y la protección de las especies objetivo de la pesca". Los resultados de este estudio revelaron que, solo la actividad pesquera por la flota de cerco dentro de la Zona Económica Exclusiva (ZEE) del Pacífico de Costa Rica, del 2002 al 2011, generó mayor extracción, estadísticamente significativa, en áreas claramente identificables tanto para el atún aleta amarilla (en sus tres clases de peso) como para las siete agrupaciones taxonómicas no objetivo de esta pesquería, que fueron analizados. En total, más de 200000 toneladas métricas de atún aleta amarilla fueron extraídas por dicha flota con fines comerciales, y más de 1300 toneladas de 49 especies de fauna distintas al atún, no objetivo de dicha flota, fueron capturadas incidentalmente durante esos diez años.

Los análisis aplicados revelaron, a un $95 \%$ de confianza, una relación diferenciada entre las variables analizadas y la captura, para cada clase de peso de atún y cada agrupación de fauna no objetivo. Sin embargo, el número de lances fue una variable explicativa de la captura estandarizada para las tres clases de peso de atún y para dos de las siete agrupaciones de fauna no objetivo.

La asociación del atún aleta amarilla pequeño (inmaduro), con la ubicación, la clorofila, la temperatura, la profundidad y los lances sobre delfines y sobre objetos flotantes, encontrada por el análisis de regresión, fue consecuente con la alta afinidad de esta clase de peso con la zona delimitada al extremo sur, evidenciada a partir del análisis de puntos calientes, así como con el alto uso de objetos flotantes como blanco para ejecutar lances en dicha zona. La afinidad del atún inmaduro con objetos flotantes, tanto naturales (i.e. troncos) como artificiales (plantados), deriva de su uso como zonas de refugio y alimentación (Jinn-Shing et al., 2015). Por eso, la ejecución de lances sobre esos objetos flotantes conlleva a alta captura, no solo de atún aleta amarilla inmaduro, sino también de una alta diversidad de especies que se congregan conformando cadenas alimentarias (Morgan, 2011; Jinn-Shing et al., 2015; Hampton et al., 2017). Posiblemente, si fueran usados objetos flotantes intensamente en otras zonas, incluido el Domo, los resultados arrojarían alta captura de atún pequeño en otras áreas de la ZZE. Es importante anotar que el uso de dispositivos agregadores de peces fue prohibido en Costa Rica desde 1999, mediante acuerdo de la Junta Directiva del Instituto Costarricense de Pesca y Acuacultura, No AJDIP/241-99.

Por su parte, la relación del atún mediano (inmaduro y maduro) con la ubicación, la clorofila y la profundidad, fue consecuente con la coincidencia espacial de esta clase de peso con el Domo Térmico, un área altamente productiva de gran extensión mar adentro y colindante con la costa, con condiciones batimétricas particulares (Fiedler, 2002). El Domo Térmico puede ser un criadero para atún aleta amarilla inmaduro en el Pacífico de Costa Rica. Freón, Guzmán y Aparicio (1997) indican que en las áreas de afloramiento costeras se encuentran los reservorios más importantes de especies pelágicas pequeñas. La presumible predominancia de individuos inmaduros dentro de esta clase de peso, de acuerdo con lo indicado en la sección Materiales y Métodos/Datos, puede ser 
posible debido a la disposición de abundantes fuentes de alimento en el domo, incluyendo larvas de cefalópodos y crustáceos y organismos zooplanctónicos, identificados dentro de la dieta de atún aleta amarilla joven (Jinn-Shing et al., 2015), y en la base de las cadenas tróficas en zonas de afloramiento (Bielecka, Krajewska-Soltys, Szymanek, \& Szymelfenig, 2005; Queiroga, Silva, Sorbe, \& Morgado, 2005). Dichas cadenas son coronadas por numerosas especies pelágicas de gran tamaño, incluido el atún aleta amarilla adulto (Hinke et al., 2004).

En el caso del atún grande (maduro), su afinidad con la ubicación, los lances sobre delfines y sobre objetos flotantes indicaron, respectivamente, fidelidad espacial a un área específica dentro del Pacífico de Costa Rica, simbiosis de atunes maduros con delfines y vinculación con objetos flotantes. Ha sido reportado que la asociación del atún maduro con delfines puede reducir el riesgo de depredación al formar grupos grandes de especies mezcladas, debido a que los atunes buscan la asociación con los delfines (Scott, Chivers, Olson, Fiedler, \& Holland, 2012). Esa relación conlleva, precisamente, a usar a esos mamíferos como blanco para ejecutar lances. Los resultados derivados de los análisis de puntos calientes mostraron una alta coincidencia entre la zona de mayor captura de atún grande y el uso de lances sobre delfines. La vinculación de atún grande con objetos flotantes, si bien no es tan común como en el caso del atún inmaduro, tampoco es rara. Los lances sobre objetos flotantes tienden a capturar atún entre 2.6 a 4.6 $\mathrm{kg}$, y los lances sobre delfines tienen a capturar atunes entre 13.5 a $37.3 \mathrm{~kg}$, pero otras combinaciones entre tipo de lance y peso también pueden darse (ITTC, 2004).

La predominancia en la captura de fauna no objetivo al sur de la ZEE revela una asociación estadísticamente significativa con la ubicación y con variables ambientales, en el caso del dorado (profundidad), el peto (latitud) y las especies diversas (latitud y profundidad). A su vez, el dorado y las especies diversas, junto con los tiburones y los picudos, están asociados a los lances sobre delfines, de manera estadísticamente significativa, de acuerdo con los análisis de regresión. Lo anterior, a pesar del alto uso de lances sobre objetos flotantes en esa área y de la influencia que dichos objetos, ya sea naturales o artificiales, pueden tener en la agregación de numerosas especies pelágicas, debido a la conformación de redes tróficas a su alrededor (Morgan, 2011; Jinn-Shing et al., 2015; Hampton et al., 2017).

La alta asociación de los lances sobre delfines con la captura incidental es esperable ya que, durante los diez años analizados, a lo largo y ancho del Pacífico de Costa Rica, los lances sobre delfines fueron utilizados con mayor frecuencia ( $\mathrm{N}=14005,84.23 \%$ ) que cualquier otro tipo de lance. Simulaciones históricas y escenarios de manejo hipotéticos han demostrado que la pesca con red de cerco afecta fuertemente los niveles tróficos más altos, conformados por especies de grandes pelági$\cos$, como las incluidas en este estudio, y que también afecta especies en niveles tróficos más bajos, como las que integran la categoría 'Especies Diversas' analizada (Hinke et al., 2004).

Finalmente, el análisis de regresión reveló una asociación estadísticamente significativa de los delfines y las mantas y rayas con la temperatura. Estas últimas también mostraron afinidad con la latitud (afinidad de sitio). Al igual que en el caso del atún mediano, la mayor captura incidental de delfines, mantas y rayas en la zona del Domo Térmico puede ser debida a alta asociación de estos grupos con dicha zona, por abundancia en la disponibilidad de alimento (Robertson \& Chivers, 1997; Navia, Mejía-Falla, \& Giraldo, 2007).

El bajo número de variables estadísticamente significativas asociadas a las capturas de las especies no objetivo, reveladas por los modelos de regresión para cada una de las agrupaciones analizadas, puede ser debido a que cada agrupación está conformada por varias especies, entonces, puede existir alta variación en las condiciones a las cuales se asocian, lo cual dificulta encontrar patrones entre las variables analizadas y la captura.

Con la información expuesta en este estudio, se brinda una perspectiva integral de la 
ZEE del Pacífico de Costa Rica para efectos de identificar zonas de mayor CPUE para el atún aleta amarilla y los grupos de otras especies pelágicas analizados, por parte de la flota de cerco entre 2002 a 2011. Se demuestra, además, la asociación entre categorías de peso de atún y los grupos de otras especies con condiciones ambientales y pesqueras particulares.

Las siguientes recomendaciones pueden ser planteadas a partir de esa información, para efectos de planificación en el uso del espacio marino en el Pacífico costarricense:

Se debe considerar la susceptibilidad al uso de red de cerco en el Domo Térmico, debido a la alta afinidad de atún mediano con esa zona (entre $2.5 \mathrm{a}<15 \mathrm{~kg}$ ), presumiblemente inmaduro en su mayoría, así como de delfines, mantas y rayas. Así mismo, deben tomar en cuenta la alta vulnerabilidad de especies de tiburones, picudos, dorado, peto y atún pequeño inmaduro, al uso de red de cerco en el sur de la ZEE.

Tanto en el Domo Térmico como en todo el sur de la ZEE, debe ser prioritario el uso de artes selectivas y, en toda la ZEE, debe ser respetada la prohibición del uso de dispositivos agregadores de peces o plantados, incluidos dentro de la categoría de objetos flotantes aquí analizada. Estas medidas serán claves para reducir la mortalidad de especies no objetivo de alto valor turístico (delfines, mantas, rayas, tiburones y especies diversas de pequeño tamaño), así como la mortalidad de especies de gran importancia tanto para pesca extractiva como turística (dorado, peto, picudos y atún inmaduro).

Resulta pertinente analizar el diseño de la zonificación de pesquerías existente para el Pacífico de Costa Rica, ya que la Zona V, la cual da acceso a la flota de cerco (MATERIALES Y MÉTODOS/Área de Estudio), traslapa significativamente con el Domo Térmico. Así mismo, es pertinente redefinir la Zona III para que abarque exclusivamente un área al Este y Sureste de la Isla del Coco, y del Área Marina de Manejo Montes Submarinos, donde se identifica la mayor captura de atún maduro, en lugar de rodearlas.
En lo referente al Domo Térmico, su alta importancia para el desarrollo de comunidades pelágicas marinas como las analizadas en este estudio, y como potencial criadero para el atún aleta amarilla, sugiere que dicha área sea manejada en forma prioritaria bajo políticas que aseguren la conservación de los procesos ecológicos que origina y mantiene, como una fuente permanente de vida y de recursos en el Pacífico de Costa Rica.

Los resultados de este trabajo son una base de conocimiento muy puntal, por estar referida a una sola flota pesquera, en un lapso de diez años. Si bien, los resultados obtenidos son de gran utilidad, no fueron realizadas comparaciones entre períodos de tiempo dentro de ese lapso, pues fraccionar los datos en períodos menores para realizar comparaciones temporales en los análisis, no habría permitido encontrar patrones, por dilución y aumento de variabilidad en la distribución espacial y la magnitud de las capturas. Una alternativa es que Costa Rica sistematice la obtención de datos de la CIAT, tanto de años previos como posteriores a los diez años analizados para este artículo, de manera que sea posible comparar entre décadas.

Los métodos aquí utilizados pueden ser aplicados también para otras flotas de pesca extractiva en Costa Rica (i.e. artesanal, mediana escala y avanzada), si para las mismas se implementan procesos sistemáticos para el levantamiento de datos pesqueros georreferenciados in situ, a largo plazo, considerando, como variables esenciales mínimas, las coordenadas de ubicación y hora de inicio y final por lance, fecha, especies objetivo y no objetivo (incidentales) extraídas y su peso total, talla, tipo de flota y arte de pesca utilizado.

Una amplia cobertura de análisis espaciales y temporales de la actividad pesquera en Costa Rica permitiría generar información para identificar zonas clave para las especies, determinar el esfuerzo pesquero aplicado sobre las mismas, conocer áreas y especies altamente explotadas, así como áreas y especies con potencial aprovechamiento sostenible, identificar zonas con alta presencia de individuos 
jóvenes (criaderos) y comprender los efectos de distintos tipos de artes de pesca sobre las capturas, tanto en términos de especies, como de grupos de edad; todo para una adecuada planificación y manejo de las actividades pesqueras, en beneficio de su sostenibilidad, desde una perspectiva ecosistémica (Abella et al., 2001; Pikitch et al., 2004; Lanz, Nevárez-Martínez, López-Martínez, \& Dworak, 2008; Moore et al., 2013; Saraux et al., 2014; Castrejón \& Charles, 2020).

Declaración de ética: los autores declaran que todos están de acuerdo con esta publicación y que han hecho aportes que justifican su autoría; que no hay conflicto de interés de ningún tipo; y que han cumplido con todos los requisitos y procedimientos éticos y legales pertinentes. Todas las fuentes de financiamiento se detallan plena y claramente en la sección de agradecimientos. El respectivo documento legal firmado se encuentra en los archivos de la revista.

\section{AGRADECIMIENTOS}

Este trabajo ha contado con el apoyo de las instituciones de afiliación de sus autores, en términos de espacio de trabajo, logística y acceso a software para análisis espaciales. Un agradecimiento especial a Eduardo Sibaja Arias, del Consejo Nacional de Rectores, a Cornelia Miller Granados, del Laboratorio PRIAS, en el Centro Nacional de Alta Tecnología, a Álvaro Morales Ramírez, del Centro de Investigación en Ciencias del Mar y a Ingo Wehrtmann, de la Unidad de Investigación Pesquera y Acuicultura, por el apoyo brindado para desarrollar esta investigación. Los datos fueron proporcionados por la Comisión Interamericana del Atún Tropical con el apoyo del Ministerio de Agricultura y Ganadería del gobierno de Costa Rica.

\section{RESUMEN}

Introducción: En los ambientes marinos del Pacífico de Costa Rica, la actividad pesquera por la flota cerquera internacional es importante debido a su amplio alcance y fuerte impacto. Objetivo: Evaluar la distribución espacial de la captura de atún aleta amarilla y especies no objetivo por esa flota, y posibles correlaciones con variables ambientales y pesqueras. Métodos: Aplicamos estadísticas geoespaciales y modelos de regresión múltiple en datos de la Comisión Interamericana del Atún Tropical, del 2002 al 2011. Utilizamos una grilla del tamaño de la Zona Económica Exclusiva (ZEE) con 1124 celdas de 22 x $22 \mathrm{~km}$ (unidad de análisis), para un total de 11240 celdas-año. Resultados: El atún mediano (presumiblemente inmaduro en su mayoría), delfines, mantas y rayas fueron capturados mayormente dentro del Domo Térmico (noreste de la ZEE). Las más altas capturas de atún pequeño (inmaduro en su totalidad), tiburones, picudos, dorado, peto y diversas especies pequeñas, ocurrieron en el extremo sur. El atún grande (maduro) fue capturado especialmente en el centro de la ZEE, hacia el este del Área Marina de Manejo Montes Submarinos y el Parque Nacional Marino Isla del Coco. Los atunes, dorado, delfines, mantas y rayas mostraron gran fidelidad de sitio por asociación con variables geográficas y ambientales, a la vez que el atún pequeño estuvo asociado a lances sobre objetos flotantes y el atún grande a lances sobre delfines. La heterogeneidad de las especies no objetivo probablemente escondió la mayoría de las correlaciones. Conclusiones: La pesca está afectando los niveles tróficos más altos y es necesario un diseño de zonificación ajustado para proteger mejor el domo térmico y considerar la susceptibilidad al uso de redes de cerco.

Palabras clave: captura incidental; atún inmaduro; análisis espacial pesquero; objetos flotantes; atún aleta amarilla.

\section{REFERENCIAS}

Abella, A., Serena, F., Fortunati, L., Mammini, U., Lari, D., \& Ciardelli, R. (2001). Use of GIS for the analysis of spatial data on fishing effort and seasonal changes in fishing strategies for Tyrrhenian Sea bottom trawl fisheries. In T. Nishida, P.J. Kailola, \& C.E. Hollingworth (Eds.), Proceedings of The First International Symposium on Geographic Information Systems (GIS) in fishery science (pp. 123-133). Japan: Fishery GIS Research Group Saitama.

Abbott, J.K., \& Haynie, A.C. (2012). What are we protecting? Fisher behavior and the unintended consequences of spatial closures as a fishery management tool. Ecological Applications, 22(3), 762-777.

Agresti, A. (2002). Categorical Data Analysis (2nd Ed.). New Jersey, USA: John Wiley \& Sons.

Arriaga, L., \& Martínez, J. (2003). Plan de ordenamiento de la pesca y acuacultura del Ecuador. Sub-secretaría de Recursos Pesqueros. Registro Oficial N 14.

Bielecka, L., Krajewska-Sołtys, A., Szymanek, L., \& Szymelfenig, M. (2005). Mesozooplankton in the Hel 
upwelling region (the Baltic Sea). Oceanological and Hydrobiological Studies, 34(2), 137-161.

Castrejón, M., \& Charles, A. (2013). Improving fisheries co-management through ecosystem-based spatial management: The Galapagos Marine Reserve. Marine Policy, 38, 235-245.

Castrejón, M., \& Charles, A. (2020). Human and climatic drivers affect spatial fishing patterns in a multiple-use marine protected area: The Galapagos Marine Reserve. PLoS ONE, 15, e0228094.

Castro, J.J., Santiago, J.A., \& Hernández-García, V. (1999). Fish associated with fish aggregation devices off the Canary Islands (Central East-Atlantic). Scientia Marina, 63(3-4), 191-198.

Courtney, F., \& Wiggin, J. (2003). Ocean Zoning for the Gulf of Maine: A Background Paper. Massachusetts, USA: Gulf of Maine Council for the Marine Environment.

Cubero-Pardo, P., \& Martínez-Cascante, D. (2013). Análisis de la Pesquería de Atún en la Zona Económica Exclusiva del Pacifico de Costa Rica. San José, Costa Rica: Federación Costarricense de Pesca.

Croll, D.A., Dewar, H., Dully, N.K., Fernando, D., Francis, M.P., Galván-Magaña, F., ... White, W.T. (2015). Vulnerabilities and fisheries impact: The uncertain future of manta and devil rays. Aquatic Conservation: Marine and Freshwater Ecosystems, 26(3), 562-575. DOI: $10.1002 /$ aqc. 2591

Davidoff, E.B. (1963). Size and year class composition of catch, age, and growth of Yellowfin Tuna in the Eastern Tropical Pacific Ocean 1951-1961. Inter-American Tropical Tuna Commission Bulletin, 8, 199-251.

Dineshbabu, A.P., Sujitha, T., Prathibha, R., \& Maheswarudu, G. (2019). Marine spatial planning for resource conservation, fisheries management and for ensuring fishermen security - Global perspectives and Indian initiatives. Current Science, 116(4), 561-567.

e Costa B.H., Gonçalves, L., \& Gonçalves, E.J. (2013). Vessels' site fidelity and spatio-temporal distribution of artisanal fisheries before the implementation of a temperate multiple-use marine protected area. Fisheries Research, 148, 27-37.

Ehler, C., \& Douvere, F. (2013). Planificación Espacial Marina. París, Francia: Comisión Oceanográfica Intergubernamental y el Programa del Hombre y la Biosfera, UNESCO.

Fiedler, P.C. (2002). The annual cycle and biological effects of the Costa Rica Dome. Deep Sea Research Part I: Oceanographic Research Papers, 49(2), 321-338.

Fonseca, A.C. (2006). Atlas marino costero de Costa Rica. En M. Quesada \& V. Nielsen (Eds.), Ambientes
Marino Costeros de Costa Rica (pp. 209-219). Costa Rica: Informe técnico para la Comisión Interdisciplinaria Marino Costera de la Zona Económica Exclusiva de Costa Rica.

Freón, P., Guzmán, R., \& Aparicio, R. (1997). Relaciones entre capturas, esfuerzo pesquero y surgencia costera en la pesquería de sardina del Oriente de Venezuela. En P. Freón, \& J. Mendoza (Eds.), La Sardina (Sardinela aurita) su medio ambiente y explotación en el Medio Oriente de Venezuela (pp. 452-471). Venezuela: Taller Cumana.

GEBCO Compilation Group. (2015). The GEBCO 2014 Grid. Recuperado de https://www.gebco.net

Great Barrier Reef Marine Park Authority (GBRMPA). (2004). Great Barrier Reef Marine Park Zoning Plan. Queensland, Australia: Great Barrier Reef Marine Park Authority.

Hall, M.A., \& Boyer, S.D. (1986). Incidental mortality of dolphins in the Eastern Tropical Pacific Tuna Fishery: Description of a new method and estimation of 1984 mortality. Report of the International Whaling Commission, 36, 375-381.

Hall, M., \& Roman, M. (2013). Bycatch and non-tuna catch in the tropical tuna purse seine fisheries of the world. FAO Fisheries and Aquaculture (Technical Paper No. 568). Roma, Italia: Food and Aquaculture Organization of the United Nations.

Hallier, J.P., \& Gaertner, D. (2008). Drifting fish aggregation devices could act as an ecological trap for tropical tuna species. Marine Ecology Progress Series, $353,255-264$.

Hampton, J., Leape, G., Nickson, A., Restrepo, V., Santiago, J., Agnew, D., ... Taquet, M. (2017). What does well-managed FAD use look like within a tropical purse seine fishery? Madrid, España: International Commission for the Conservation of Atlantic Tunas.

Hinke, J.T., Kaplan, I.C., Aydin, K., Watters, G.M., Olson, R.J., \& Kitchell, J.F. (2004). Visualizing the FoodWeb Effects of Fishing for Tunas in the Pacific Ocean. Ecology and Society, 9(1), 10-34.

Inter-American Tropical Tuna Commission (ITTC). (2004). Tunas and billfishes in the eastern Pacific Ocean in 2003. California, USA: Inter-American Tropical Tuna Commission.

Jalali, M.A., Lerodiaconou, D., Gorfine, H., Monk, J., \& Rattray, A. (2015). Exploring spatiotemporal trends in commercial fishing effort of an abalone fishing zone: a GIS-based hotspot model. PLoS One, 10(5), e0122995. DOI: 10.1371/journal.pone.0122995

Jiménez, J. (2013). Problemática marino costera en Costa Rica y necesidad de planificación. Ambientico, 250, 4-12. 
Jinn-Shing, W., Ming-An, L., Kwang-Ming, L., MingShu, H., Mine-Kune, H., \& Long-Jing, W. (2015). Feeding ecology of juvenile yellowfin tuna from waters Southwest of Taiwan inferred from stomach contents and stable isotope analysis. Marine and Coastal Fisheries, 7(1), 537-548. DOI: 10.1080/19425120.2015.1094157

Klein, C.J., Steinback, C., Watts, M., Scholz, A.J., \& Possingham, H.P. (2009). Spatial marine zoning for fisheries and conservation. Research Communication. Frontiers in Ecology and the Environment, 8(7), 349-353.

Lanz, E., Nevárez-Martínez, N.O., López-Martínez, J., \& Dworak, J.A. (2008). Spatial distribution and species composition of small pelagic fishes in the Gulf of California. Revista de Biología Tropical, 56(2), 575-590.

Lezama-Ochoa, N., Murua, H., Chust, G., Van Loon, E., Ruiz, J., Hall, M., ... Villarino, E. (2016). Present and future potential habitat distribution of Carcharhinus falciformis and Canthidermis maculata by-catch species in the tropical tuna purse-seine fishery under climate change. Frontiers in Marine Science, 3, 1-34.

Lezama-Ochoa, N., Hall, M., Román, M., \& Vogel, N. (2019). Spatial and temporal distribution of mobulid ray species in the eastern Pacific Ocean ascertained from observer data from the tropical tuna purse-seine fishery. Environmental Biology of Fishes, 102(1), $1-17$.

Meaden, G.J., Aguilar-Manjarrez, J., Corner, R.A., O'Hagan, A.M., \& Cardia, F. (2016). Marine spatial planning for enhanced fisheries and aquaculture sustainability. Its application in the Near East. Roma, Italia: Food and Agriculture Organization of the United Nations.

Mendenhall, W. (1990). Mathematical Statistics with Applications. Massachusetts, USA: PWS-KENT Publishing Company.

Minte-Vera, C.V., Maunder, N. \& Aires-da-Silva, A. (2018). Condición del atún aleta amarilla en el Océano Pacífico Oriental en 2017 y perspectivas para el futuro (Documento SAC-09-06). En 9na Reunión Comité Científico Asesor. Estados Unidos de América: Comisión Interamericana del Atún Tropical.

Moore, J.E., Curtis, K.A., Lewison, R.L., Dillingham, P.W., Cope, J.M., Fordham, S.V., ... Zhou, S. (2013). Evaluating sustainability of fisheries bycatch mortality for marine megafauna: A review of conservation reference points for data-limited populations. Environmental Conservation, 40(4), 329-344.

Moran, P.A.P. (1950). Notes on continuous stochastic phenomena. Biometrika, 37, 17-23.
Morgan, A.C. (2011). Dispositivos agregadores de peces (plantados) y atún. Impactos y opciones de ordenación. Washington, USA: Ocean Science Division, The PEW Environmental Group.

Navia, A.F., Mejía-Falla, P.A., \& Giraldo, A. (2007). Feeding ecology of elasmobranch fishes in coastal waters of the Colombian Eastern Tropical Pacific. BMC Ecology, 7(8), 1-10. DOI:10.1186/1472-6785-7-8

OBPG. (2020). Moderate Resolution Imaging Spectroradiometer (MODIS) Aqua Level L3 Yearly $4 \mathrm{~km}$ Daytime SST and Chlor. Ocean Color Data, NASA OB. DAAC, Greenbelt. Recuperado de https://oceancolor.gsfc.nasa.gov

Ord, J.K., \& Getis, A. (1995). Local spatial autocorrelation statistics: distributional issues and an application. Geograpjical Analysis, 27, 286-306.

Ord, J.K., \& Getis, A. (2001). Testing for local spatial autocorrelation in the presence of global autocorrelation. Journal of Regional Science, 41, 411-432.

Paxinos, R., Wright, A., Day, V., Emmett, J., Frankiewicz, D., \& Goecker, M. (2008). Marine Spatial Planning: Ecosystem-based zoning methodology for marine management in South Australia. Journal of Conservation Planning, 4, 37-59.

Pikitch, E.K., Santora, C., Babcock, E.A., Bakun, A., Bonfil, R., Conover, D.O., ... Sainsbury, K.J. (2004). Ecosystem-Based Fishery Management. Science, 305, 346-347.

Queiroga, H., Silva, C., Sorbe, J.C., \& Morgado, F. (2005). Composition and distribution of zooplankton across an upwelling front on the Northern Portuguese coast during summer. Hydrobiologia, 545, 195-207. DOI: 10.1007/s10750-005-2675-y

Riolo, F. (2006). A geographic information system for fisheries management in American Samoa. Environmental Modelling \& Software, 21(7), 1025-1041.

Robertson, K.M., \& Chivers, S.J. (1997). Prey occurrence in pantropical spotted dolphins, Stenella attenuata, from the eastern tropical Pacific. Fishery Bulletin, 95, 334-348.

Scott, M.D., Chivers, S.J., Olson, R.J., Fiedler, P.C., \& Holland, K. (2012). Pelagic predator associations: tuna and dolphins in the eastern tropical Pacific Ocean. Marine Ecology Progress Series, 458, 283302. DOI: $10.3354 /$ meps09740

Safina, C. (1993). Bluefin Tuna in the West Atlantic: Negligent Management and the Making of an Endangered Species. Conservation Biology, 7(2), 229-234.

Salas, E, Salazar, E.R., \& Arias, A. (2012). Diagnóstico de Áreas Marinas Protegidas y Áreas Marinas para la 
Pesca Responsable en el Pacifico costarricense. San José, Costa Rica: Fundación MarViva.

Sanchirico, J.N. (2000). Marine Protected Areas as Fishery Policy: A Discussion of Potential Costs and Benefits. Washington, USA: Resources for the Future.

Saraux, C., Fromentin, J.M., Bigot, J.L., Bourdeix, J.H., Morfin, M., Roos, D., ... Bez, N. (2014). Spatial Structure and Distribution of Small Pelagic Fish in the Northwestern Mediterranean Sea. PLoS ONE, 9(11), e111211. DOI: 10.1371/journal.pone.0111211
Torres-Irineo, E., Amandè, M.J., Gaertner, D., Delgado, A., Murua, H., Chavance, P., ... Lezama-Ochoa, N. (2014). Bycatch species composition over time by tuna purse-seine fishery in the eastern tropical Atlantic Ocean. Biodiversity and Conservation, 23, 1157-1173.

Watters, G. M., \& Maunder, M. N. (2001). Status of bigeye tuna in the eastern Pacific Ocean. Inter-American Tropical Tuna Commission, Stock Assessment Report, $1,109-211$

See Digital Appendix at: / Ver Apéndice digital en: revistas.ucr.ac.cr 